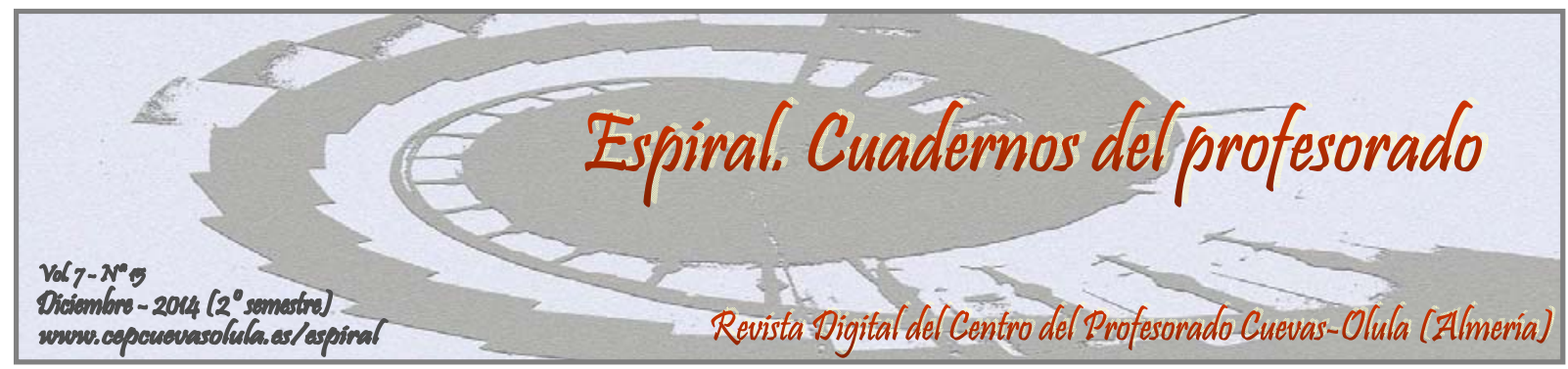

\title{
ACTIVIDAD FÍSICO-EDUCATIVA EN EL MEDIO NATURAL: INFLUENCIA DE PRÁCTICA FÍSICA SOBRE LA CONDICIÓN FÍSICA EN MUJERES MAYORES
}

\author{
PHYSICAL EDUCATION ACTIVITY IN THE NATURAL ENVIRONMENT: \\ INFLUENCE OF PRACTICE ON PHYSICAL FITNESS IN OLD WOMEN
}

\author{
Pedro J. Ruiz Montero ${ }^{(1)}$, Antonio Baena-Extremera ${ }^{(2)}$ \\ y José David Ayala Martínez ${ }^{(2)}$
}

(1) Facultad de Ciencias de la Actividad Física y del Deporte, Universidad de Granada, España

(2) Facultad de Ciencias del Deporte, Universidad de Murcia, España

(3) Universidad Europea de Madrid, España

RESUMEN: Las actividades físicas en el medio natural han experimentado un auge considerable en la última década hasta el día de hoy. A esto hay que sumarle la función de distensión que puede producir en personas inmersas en las imposiciones actuales de nuestra sociedad. La muestra del presente estudio se compone de 43 mujeres, todas ellas participantes del programa de Actividad Física para Mayores de la provincia de Málaga, con edades comprendidas entre los 60 y 79 años y que pertenecen a tres poblaciones inferiores a 3.000 habitantes, en una comarca denominada Sierra de las Nieves. Las participantes cumplimentaron un cuestionario sociodemográfico sobre la participación en el citado programa y realizaron todas las pruebas físicas de las que se compone el Senior Fitness Test (Rikli and Jones, 1999). Los resultados obtenidos muestran diversas correlaciones negativas entre diferentes variables de la condición física de las participantes con determinadas características sociodemográficas y muy pocas relaciones positivas entre las mismas variables.

Palabras clave: condición física; medio natural, personas mayores, mujeres.

ABSTRACT: Physical activities in the natural environment have experienced a considerable growth in the last decade until today. To this we must add the function of relaxation that can occur in people immersed in the current constraints of our society. The sample of this study consists of 43 women, all program participants Physical Activity for Older province of Málaga, aged between 60 and 79 years and belongs to three populations less than 3.000 inhabitants, in a region called Sierra de las Nieves. The participants completed a sociodemographic questionnaire on participation in that program and performed all the physical tests which include the Senior Fitness Test (Rikli and Jones, 1999). The results show various negative correlations between different variables of the physical condition of the participants with certain sociodemographic characteristics and very few positive relationships between those variables.

Key words: fitness; environment, seniors, women. 
Ruiz Montero, P. J., Baena-Extremera, A., y Ayala Martínez, J. D. (2014). Actividad físicoeducativa en el medio natural: influencia de práctica física sobre la condición física en mujeres mayores. Espiral. Cuadernos del Profesorado, 7(15), 55-63. Disponible en: http://www.cepcuevasolula.es/espiral

Fecha de recepción: 05/03/2014

Fecha de aceptación: 22/06/2014
Enviar correspondencia a: abaenaextrem@um.es

\section{1.- INTRODUCCIÓN}

Las actividades físico-deportivas que se desarrollan el medio natural y están relacionadas con este, han experimentado un crecimiento considerable en la población española. Este tipo de práctica física se ha convertido en una cuestión de necesidad al entenderse como medio de liberación de cargas impuestas por la sociedad actual (García Ferrando, 2006).

Las actividades físicas, educativas o deportivas en la naturaleza son entendidas según Parra (2001) como prácticas motrices con un componente físico y educativo con común denominador: la Naturaleza. A su vez, también hay un carácter lúdico y recreativo entre otras, donde el individuo se desarrolla de forma global e integral, creándose una conciencia ecológica. Todo ello, debido a que el aire libre constituye un marco privilegiado para la intervención pedagógica en personas de cualquier edad (Granero y Baena, 2007).

Mantener un estilo de vida saludable es muy importante a lo largo de toda la vida, siendo el medio natural un recurso fácil al alcance de muchas personas con edad adulta o mayor. Los hábitos saludables pueden reducir riesgos como lesiones, enfermedades crónicas, cardiovasculares y neurológicas en la tercera edad. Por consiguiente, todo ello influirá de manera satisfactoria en una mejora de la calidad de vida y mayor longevidad (Akesson et al., 2007). Si el medio escogido para esta mejora del estilo de vida es el entorno natural, la naturaleza favorecerá una actitud de respeto y comprensión hacia los elementos que conforman un ecosistema determinado, presentando este aspecto una larga tradición en Estados Unidos (Neill, 2004).

En cuanto al papel de las personas mayores en la actualidad, decir que a nivel mundial, es el segmento poblacional que más crecimiento está experimentando (Jenkins, 2005). Un papel importante en todo este proceso lo protagoniza la figura de la mujer, donde el valor medio será aún mayor que el de hombres (100 mujeres por cada 78 hombres). Esta causa es una esperanza de vida superior al de los hombres, implicando ello una paridad en cuanto el problema del envejecimiento mundial y su tratamiento político y legislativo (Ruiz-Montero y Baena, 2009). La población femenina española también supera a la masculina en medio millón de personas aproximadamente. Esto se traduce en 23.264.850 mujeres respecto a 22.798.661 hombres, según datos del Instituto Nacional de Estadística (INE, 2008). Ramos y Pinto (2005) vaticinan un crecimiento considerable de la población mayor española a causa de los positivos hábitos de vida saludables y calidad de vida.

Un factor que comporta una variedad de beneficios en la salud personal es la práctica de ejercicio físico (Endres, 2003), haciendo énfasis en la mejora de la calidad de vida y mayor longevidad de las personas, todo ello mediante la práctica de ejercicio variado y su respectiva adaptación a las capacidades personales de las propias personas (Rosenfeld y Tenembaum, 1992).

El objetivo de este estudio ha sido analizar el nivel de condición física de mujeres que pertenecen a un programa de condición física enfocado a personas mayores, con edades comprendidas entre los 60 y 76 años. Para ello, se ha realizado una evaluación física a través de una batería estandarizada de ejercicios con el fin de establecer una relación entre la condición física de las participantes y sus características sociodemográficas, así como hábitos de práctica física. 


\section{2.- MATERIAL Y MÉTODO}

El presente trabajo se centra en una investigación con un diseño seleccional, descriptivo y correlacional de las variables sociodemográficas que se muestran respecto a la calidad de vida relacionada con la salud y niveles de capacidad funcional.

\section{Muestra}

Se evaluaron a 43 mujeres con edades comprendidas entre 60 años en adelante, que de forma voluntaria formaron parte del programa de actividad físico deportiva para mayores de la Diputación Provincial de Málaga, dirigida a este sector poblacional en diferentes municipios ubicados en la provincia de Málaga. Las características específicas se presentan en la Tabla I.

Tabla 1. Parámetros sociodemográficos de las participantes

\begin{tabular}{|lcccc|}
\hline Parámetro (n= 43) & Mínimo & Máximo & Media & SD \\
Edad & 60 & 77 & 66.34 & 5.26 \\
Peso (kg) & 49.90 & 102.80 & 72.30 & 12.01 \\
Talla (m) & 1.41 & 1.60 & 1.52 & .044 \\
IMC & 22.48 & 42.79 & 31.14 & 4.74 \\
\hline
\end{tabular}

Los tres municipios de donde se han obtenido, están ubicadas geográficamente en la Sierra de las Nieves (al noroeste de la provincia de Málaga).

\section{Instrumentos}

Se utilizó un cuestionario sociodemográfico, con preguntas de tipo categorizadas y con diferentes opciones a elegir una a excepción de dos, que son abiertas.

Para la valoración de la condición física funcional con personas mayores, una batería de test diseñada específicamente para la población mayor es el Señor Fitness Test (SFT) de Rikli y Jones (1999). Los principales aspectos a la hora de confeccionar estas pruebas fueron la facilidad ejecución y realización en cualquier lugar, siempre con el debido rigor científico en función de su fiabilidad y validez (Rikli y Jones, 2001). Las diferentes pruebas evalúan la fuerza y flexibilidad del tren superior e inferior, la capacidad aeróbica y la agilidad-equilibrio.

Para el desarrollo de esta batería de pruebas, se han utilizado criterios tales como:

- Reflejo de cambios en el rendimiento físico relacionados con la edad.

- Capacidad para detectar cambios producidos por el ejercicio físico o entrenamiento.

- Fácil de administrar y puntuar.

- Rápidos en la puesta en práctica.

- Etc.

Si nos referimos a calidad de vida, el cuestionario SF-36 en su versión castellana (Alonso et al., 1995) es una escala genérica que posibilita el conocimiento de los estados positivos y negativos de la salud física y psicológica, siendo aplicable a pacientes y a la población en general. Su utilidad a la hora de relacionar la calidad de vida con la salud, comparando el peso que tiene las enfermedades en la salud y los beneficios producidos por diferentes aspectos, son de gran relevancia para cualquier estudio e investigación. Todo esto sirve para valorar el estado de salud de pacientes individuales (Ware, 2000). Este instrumento se compone de 36 ítems temporalizados en un periodo estándar de cuatro semanas, que abordan las nueve escalas/ dimensiones del estado de salud utilizadas con más frecuencia en los principales cuestionarios de salud. Las nueve dimensiones son función física, rol físico, dolor corporal, salud general, vitalidad, función social, rol emocional, salud mental y evolución de la salud. Decir que este instrumento alberga un componente físico (función física, rol físico, dolor corporal y salud en general) y otro mental (salud mental, rol emocional, función social y vitalidad).

\section{Procedimiento}


En primer lugar se seleccionó a las participantes del estudio, integrantes del programa de condición física de mayores de la Diputación de Málaga y que pertenecen a municipios adscritos a dicho programa.

La estrategia utilizada a la hora de aplicar los cuestionarios fue mediante contacto directo con las mujeres de la muestra, aclarando posibles dudas y explicando con claridad cada una de las pruebas y cuestionarios. Todas las participantes del estudio fueron informadas de la confidencialidad de las informaciones y prueba de ello es la hoja de consentimiento firmada de manera voluntaria.

Las pruebas físicas se realizaron dos días después de la última sesión semanal, de manera individual a excepción de "andar 6 minutos" donde se llevaba a cabo por parejas, posteriormente se pasaría a la cumplimentación de cuestionarios mediante entrevista (contacto personal). Las participantes eran informadas al comenzar a utilizar cada instrumento, solventando posibles dudas y recordando normas de seguridad y prevención de riesgos.

Las participantes de este estudio eran seleccionadas de entre las asistentes regularmente a clases de condición física, cumpliendo con el requisito de ser mayores de 60 años, puesto que es la edad mínima requerida para participar en el programa de la Diputación de Málaga. Ninguna de ella podría tener patologías físicas y psicológicas graves que pudiesen afectar negativamente en la salud propia, ni comer o fumar como mínimo una hora antes de la realización de las pruebas.

\section{Análisis estadístico}

Para el tratamiento de los datos del presente estudio, se ha aplicado un análisis descriptivo de la muestra $(n=43)$ en cuanto las pruebas que componen el $S F T$, utilizándose la media, desviación típica, máximo y mínimo.

Tras la tabulación y mecanización de los datos, el análisis de los mismos se ha tratado mediante SPSS versión 17 para WINDOWS, programa que se ha seleccionado por su orientación en el campo sociológico (Scientific Packet Social Statistic), posibilitando una puesta en práctica de diferentes técnicas estadísticas precisas para nuestro estudio. En primer lugar se realizaron análisis de normalidad (prueba de Kolmogórov-Smirnov), resultando la variable condición física paramétrica y calidad de vida no paramétrica. Posteriormente, se realizaron análisis descriptivos en función del de la edad, peso e IMC. Se realizó una prueba T de Kendall para relacionar las escalas que componen la variable calidad de vida con los diferentes ejercicios del SFT (Rikli y Jones, 1999) $(p \leq 0.01 ; p \leq 0.05)$.

\section{3.- RESULTADOS}

En relación a las variables tratadas dentro del grupo denominado composición corporal, la edad es interesante ya que presenta un valor mínimo de 60 años (condición indispensable para pertenecer al programa de mayores de la Diputación de Málaga) y un máximo de 77 (personas con más edad que ha participado en el presente estudio). La media es de 66.34+ 5.26 años. El peso medio de las participantes del presente estudio es de $72.30+12.01 \mathrm{~kg}$, mientras que la talla proporciona valores medios de 1.52+ $0.044 \mathrm{~m}$. Relacionada con ambas está el IMC, con un mínimo de 22.48 y máximo de 42.79. La media de esta variable es 31.14+ 4.74 (Tabla 1).

La Tabla 2 refleja la batería de ejercicios que conforma la evaluación de la capacidad funcional. El SFT.1 es la prueba en la que se cuenta las veces que se levanta y se siente una persona en una silla, siendo la media baja. Sin embargo, la fuerza del tren superior, mediante la flexión de brazos con una pesa de 2. $27 \mathrm{~kg}$, presenta valores acordes a la media de edad de las participantes. La prueba de flexión de tronco hacia delante también presenta valores medios. Por el contrario, la flexión de tren superior mediante el alcance de las manos por la espala presenta valores bajos. La capacidad aeróbica también es baja debido a que los metros recorridos durante seis minutos no corresponden a la distancia media para mujeres comprendidas entre los 65-59 años (500-635 metros). La última variable de esta batería es el SFT.6, conocido como la prueba de levantarse, rodear un cono y volverse a sentar, obteniéndose una media adecuada a la media de edad de las participantes. 
Tabla 2. Resultados de las pruebas que componen el SFT

\begin{tabular}{|lcccc|}
\hline Variable (n= 43) & Mínimo & Máximo & Media & SD \\
$\begin{array}{l}\text { SFT.1. Sentarse y levantarse } \\
\text { de una silla }\end{array}$ & 6 & 16 & 10.74 & 2.22 \\
SFT.2. Flexiones de brazo & 10 & 18 & 14.79 & 1.97 \\
$\begin{array}{l}\text { SFT.3. Sentado y alcanzar el } \\
\text { pie extendido }\end{array}$ & -22 & 24 & -0.39 & 8.35 \\
$\begin{array}{l}\text { SFT.4. Juntar las manos } \\
\text { tras la espalda }\end{array}$ & -28 & 8 & -6.67 & 9.29 \\
$\begin{array}{l}\text { SFT.5. Caminar 6 minutos } \\
\text { SFT.6. Levantarse, caminar y }\end{array}$ & 228.5 & 685.5 & 476.39 & 86.20 \\
volverse a sentar & 4 & 10.70 & 6.02 & 1.28 \\
\hline
\end{tabular}

Los resultados obtenidos en la variable calidad de vida, presentan medias de puntuación elevadas en sus ochos escalas, a excepción de la evolución de la salud que no ha sido incluida debido a su singularidad (solo es un ítem) (Tabla 3).

Tabla 3. Valores de las escalas que componen el SF-36

\begin{tabular}{lcccc|} 
Escala & Mínimo & Máximo & Media & SD \\
\hline Función Física & 0 & 100 & 74.06 & 21.22 \\
Rol Físico & 0 & 100 & 70.93 & 35.32 \\
Dolor Corporal & 22 & 100 & 65.27 & 23.60 \\
Salud General & 5 & 100 & 61.83 & 20.56 \\
Vitalidad & 0 & 95 & 59.76 & 20.61 \\
Función Social & 37.5 & 100 & 82.55 & 19.12 \\
Rol Emocional & 0 & 100 & 78.29 & 34.02 \\
Salud Mental & 24 & 100 & 67.53 & 19.56 \\
\hline
\end{tabular}

Las ocho escalas del SF-36 se correlacionan con las seis pruebas del SFT, estableciéndose una mayor significatividad en la variable SFT.1, sentarse y levantarse (fuerza tren superior) con el resto de variables, la escala de dolor corporal y salud mental, aunque otras muchas variables son significativas con otras variables. La prueba de flexión de brazos presenta asociación significativa con la función física, dolor corporal y salud general. De las dos pruebas que evalúan la flexibilidad, solo el SFT.4, juntar las manos tras la espalda tiene significatividad con una escala, concretamente la salud mental. La prueba de caminar 6 minutos (SFT.5) conlleva una correlación significativa con las escalas función física y rol físico, dolor corporal y salud general. El ejercicio de levantarse, caminar y volverse a sentar es inversamente proporcional a las escalas del SF-36 función física, rol físico, dolor corporal y salud mental (Tabla 4). 
Tabla 4. Relación entre escalas del SF-36 y la batería de ejercicios del SFT

\begin{tabular}{|c|c|c|c|c|c|c|}
\hline 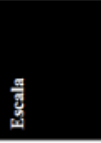 & 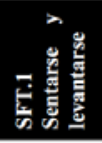 & 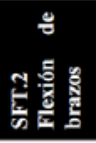 & 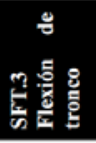 & 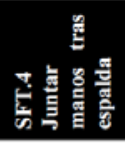 & 恕咅 & 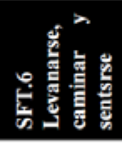 \\
\hline F. Fisica & $.379^{\circ 60}$ & $.232^{\circ}$ & .062 & .029 & $.258^{*}$ & $-.317^{* 4 \%}$ \\
\hline R. Físico & $.371^{\circ *}$ & .237 & .115 & .063 & $.289^{\circ}$ & $-.248^{\circ}$ \\
\hline Dolor C. & $.506^{* *}$ & $.309^{* *}$ & -.062 & .058 & $.328^{* *}$ & $-366^{* *}$ \\
\hline Salud G. & $.255^{\circ}$ & $.270^{*}$ & .040 & .171 & $.311^{\circ \%}$ & -167 \\
\hline Vitalidad & $.392^{\text {* }}$ & .219 & .022 & .205 & .209 & $-.330^{6 \omega}$ \\
\hline F. Social & $.248^{\circ}$ & .141 & .085 & .066 & .235 & -.172 \\
\hline Rol Emoc. & c. $271^{*}$ & .121 & .181 & .172 & .239 & -.172 \\
\hline Salud M. & $.320^{* *}$ & .180 & .142 & $.295^{* *}$ & .188 & $-293^{* *}$ \\
\hline
\end{tabular}

${ }^{* *} p \leq 0.01 ;{ }^{*} p \leq 0.05$

\section{4.- DISCUSIÓN Y CONCLUSIONES}

El objetivo de este estudio fue evaluar las mediciones antropométricas, de condición física y calidad de vida relacionada con la salud, tras un mes de actividades físicas aeróbicas realizadas en la naturaleza y que forman parte del programa de Condición Física de la Diputación Provincial de Málaga.

La práctica de actividad física de manera regular, es un factor que determina la calidad de vida de las personas, teniendo como repercusión principal beneficios psicológicos (Blasco, Capdevilla, y Cruz, 1994). Además, el carácter cambiante de la naturaleza va a convertirse en el principal exponente del desarrollo de las actividades físicas desarrolladas en ella. Precisamente, va a ser la incertidumbre asociada a sus imprevisibles cambios, el principal rasgo que define y diferencia a las actividades físico-educativas desarrolladas en el marco natural (Baena, 2006).

Los resultados obtenidos en el presente estudio muestran como la composición corporal de las participantes presentan valores altos (IMC $=31.14 \mathrm{Kg} / \mathrm{m} 2 \pm 4.74)$ y un elevado peso medio $(72.30 \mathrm{Kg}$ +12.01). Según la OMS (1998), el valor medio de las participantes se engloba dentro del rango obesidad tipo I, con valores que oscilan entre 30.0 y $34.9 \mathrm{Kg} / \mathrm{m} 2$.

Los resultados obtenidos en la realización de esta batería de ejercicios, muestran una valoración diversa en cuanto a capacidad física. Determinadas pruebas se encuentran por debajo del rango de puntuación propio a su edad pero no otras. La prueba relativa a fuerza de tren inferior, en comparación con un grupo de 22 mujeres y utilizando una metodología muy similar a la de este trabajo, presenta un valor medio de 14.86+1.98 (Garatachea et al., 2004). Por consiguiente es mayor que el valor medio obtenido en el presente estudio. El valor medio obtenido para la prueba de fuerza de tren superior (brazos) es de 14.79 1.97 repeticiones como media de los dos brazos, estando por debajo de los valores obtenidos en otros estudios como los llevado a cabo por Marques (2002). Este último estudio, con una media pre-post test de 16.90 repeticiones para una muestra femenina portugués ó 19.25 repeticiones de una muestra de 357 mujeres mayores del Algarve portugués (Martín Rodríguez, 2006).

La muestra portuguesa antes citada por Marques (2002) establece un valor medio de 3.00+ $9.85 \mathrm{~cm}$ pre-test y $2.57 \pm 7.81 \mathrm{~cm}$ pos-test para la flexibilidad del tren inferior, mientras que nuestra muestra establece un valor medio de -0.39 . No obstante, aunque es menor que los valores de la muestra portuguesa, están recogidos dentro de los parámetros normales para esta prueba y para la media de edad de las participantes. De la misma manera, la media obtenida en la flexibilidad del tren inferior para la muestra Del presente estudio es también negativa, al igual que la anterior, concretamente $6.67 \pm 9.29 \mathrm{~cm}$. Este valor la ubica en el rango de puntuación de edades comprendidas entre los 85 y 89 años. Por su parte, el estudio de Martín Rodríguez ya citado anteriormente, presenta 
un valor pre-test de $-8.7 \pm 11.2 \mathrm{~cm}$ y post test de $-7.4 \pm 10.9 \mathrm{~cm}$. Ambos valores son mayores negativamente, lo que significa que las mujeres de la presente investigación presentan una mejor flexibilidad de tren superior

Un ejercicio práctico para evaluar el equilibrio de las personas mayores es la prueba de levantarse, caminar y volverse a sentar. El valor obtenido en la presente muestra es de $6.02 \pm 1.28$ seg., situándose en el rango de puntuación de edades comprendidas entre los 60 y 64 años. Como referencia podemos destacar los valores obtenidos en diversos estudios, ubicados todos ellos en una localización temporal de medio segundo por debajo del valor de la presente investigación. Cepero et al. (2009) obtuvieron un valor de 5.04+ 0.9 seg., siendo menor respecto al tiempo obtenido en el presente estudio. Marques (2002) y su estudio con 60 personas (46 de ellas mujeres) también obtuvo un valor menor que la presente investigación (5.30seg.), con una muestra que presentaba edades muy similares $(68.25 \pm 5.05)$ a la del presente estudio.

La última capacidad física evaluada fue la resistencia aeróbica. La muestra del presente estudio obtuvo un valor medio de 476.39+ $86.20 \mathrm{~m}$, encontrándose en la media de rango de puntuación para mujeres con edades comprendidas entre 70-74 años. Esta distancia es próxima a un estudio elaborado con otra muestra española (Morales et al., 2013), donde se comparaban mujeres mayores de 65 años sedentarias y no sedentarias. Los resultados obtenidos por las mujeres sedentarias fue de $535.5 \pm 73.7 \mathrm{~m}$ en mujeres no sedentarias y de $502.5 \pm 102.5 \mathrm{~m}$ para las sedentarias. Por consiguiente, la presente muestra estaría cercana a las mujeres sedentarias de la muestra de Morales et a., con la única diferencia que las participantes de este estudio si realizan ejercicio físico dos veces por semana.

En cuanto la calidad de vida de las presentes participantes, decir que el componente físico presenta en su cuatro dimensiones, valores más altos que los de otros estudios como son los de Domínguez et al. (2009) (rol físico=69+41), Marques (2002) (dolor corporal=65.27+23.60). Al referirse al componente mental, los resultados obtenidos en la presente muestra también presentan valores más altos que los de otra muestra de Pinto et al. (2009) (vitalidad=37.3+20.4) o similares al del citado estudio de Domínguez et al. (función social=82.91_23.67).

Todas las pruebas que conforman el Senior Fitness Test muestran algún tipo de diferencia significativa. Algunas de ellas mediante correlaciones inversas, a excepción de la prueba 3, estar sentado y alcanzar el pie en extensión. Todo lo contrario es la prueba de sentarse y levantarse de una silla, en la que se puede observar una correlación significativa respecto a todas las escalas del SF-36. Esta prueba evalúa el tren inferior, mientras que la prueba de flexión de brazo evalúa el tren superior. Ambos ejercicios o pruebas, muestran una asociación significativa con las escalas de función física, dolor corporal y salud general. Esto coincide con diferentes estudios en los que se muestra como la práctica de actividad física mediante ejercicios de fuerza y mantenimiento muscular, acompañándose de una continuidad en el tiempo, afectan positivamente en los estados de ánimo enérgicos y de vigor de manera considerable (Schwerdtfeger, Eberhardt, y Chmitorz, 2008), afectando esto directamente a la significatividad establecida entre fuerza del tren inferior y la escala vitalidad. Ruiz-Montero y Baena (2009) destacan en un estudio sobre fuerza prensil manual en mujeres mayores de 60 años, como una actividad de sostenimiento muscular obtiene diferencias significativas entre la práctica física como mejora de la salud con el estado de vigor.

La prueba de flexión del tren inferior (estar sentado y con la pierna en extensión tocarse el pie) no se muestra significatividad alguna y para la flexión del tren superior o prueba de juntar las manos por detrás, solo se produce correlación significativa con la salud mental. Es difícil encontrar estudios que relacionen la práctica física a través de la flexibilidad con el bienestar psicológico. En cuanto la prueba que evalúa la capacidad aeróbica, se aprecia significatividad con las escalas físicas (función y rol físico), dolor corporal y salud general. Esta correlación presenta similitud con los estudios que corroboran como las pruebas evaluativas de capacidad aeróbica, que consisten en andar/ caminar durante pocos espacios de tiempo, se asocia con diferentes estados de salud (Bittner et al., 1993). 


\section{5.- REFERENCIAS}

Akesson, A., Weismayer, C., Newby, P.K., y Wolk, A. (2007). Combined effect of lowrisk dietary and lifestyle behaviors in primary prevention of myocardical infarction in women. Archives of internal medicine, 19(167), 2122-2127.

Alonso, J., Prieto, L., y Antó, J.M. (1995). La versión española del SF36 Health Survey (Cuestionario de Salud SF-36): un instrumento para la medida de los resultados clínicos. Medicina Clínica, 104 (20), 771-776.

Baena, A. (2006). Actividades en el medio natural. Tipos, clasificaciones y recursos. Organización de actividades física en la naturaleza. Granada: Inédito.

Bittner, V., Weiner, D., Yusuf, S., Rogers, W., McInty, K.M., Bangdiwala, S.I. et al. (1993). Prediction of martality and morbidity with a 6-minute walk test in patients with left ventricular dysfunction. Journal of the American Medical Association, 270, 1702-1707.

Blasco, T., Capdevila, LL., y Cruz, J. (1994). Relaciones entre actividad física y salud. Anuario de psicología, 6 (12), 19-24.

Domínguez, A., Sánchez, D., García, A., Escalante, Y., y Saavedra, J.M. (2009). Influencia de un programa de acondicionamiento físico sobre la calidad de vida relacionada con la salud en mujeres mayores sedentarias. En Montiel, P., Merino, A., Sánchez, A., Heredia, A., y Salinas, F. (comp.), III Congreso Internacional de Actividad Físico Deportivo para mayores (pp. 887- 892). Málaga: CEDMA.

Endres, M., Gertz, K., Lindauer, U., Katchanov, J., Schultze, J., y Schröck, H., (2003). Mechanisms of stroke protection by physical activity. Annals of Neurology, 54, 582-90.

Garatachea, N., Val Ferrer, R., Calvo, I., y de Paz, J.A. (2004). Valoración de la condición física funcional, mediante el Senior Fitness Test, de un grupo de personas mayores que realizan un programa de actividad física. Apunts Medicina de l'Esport, 76, 22-26.

García Ferrando, M. (2006). Veinticinco años de análisis del comportamiento deportivo de la población española (1980-2005). Revista Internacional de Sociología, 44, 15-38.

Granero, A. y Baena, A. (2007). Importancia de los valores educativos de las actividades físicas en la naturaleza. Habilidad Motriz, 29, 5-14.

INE (2014). Demografía y población española, 2008. Instituto Nacional de Estadística; España.

Jenkins, C.D. (2005). Mejoremos la salud a todas las edades. Un manual para el cambio de comportamiento. Washington: Panamericana Health Organization.

Marques, R.M. (2002). Efeitos da Prática da Actividade Física sobre a Aptidao Física de Adultos Idosos. Tesis Doctoral, Universidad de Oporto, Oporto.

Martín Rodríguez, M. (2006). Influencia de un programa de actividad física sobre aspectos físicos y psicológicos en personas de más de 55 años en la población del Algarbe. Tesis Doctoral, Universidad de Valencia, Valencia.

Matsudo, S. (1992). Perscripcao e beneficios da actividade física na terceira idade. Revista Brasileira Ciencias del Movimineto, 4, 19-30.

Molinero, F., Río, J., López, A., Prados, P., Fernández, I. y Martínez, A. (2009). En Montiel, P., Merino, A., Sánchez, A., Heredia, A. y Salinas, F. (comp.), III Congreso Internacional de Actividad Físico Deportivo para mayores (pp. 505- 507). Málaga: CEDMA.

Morales, S; Gómez-Cabello, A; González-Agüero, A; Casajús, JA; Ara J y Vicente-Rodríguez, G. Sedentarismo y condición física en mujeres postmenopáusicas. Nutr Hosp 2013, (28)3: 1053-59.

Neill, J.T. (2004). Experiential learning cycles [en línea]. Disponible en: http://www.wilderdom.com/theory/ExperientialL earningCycles.html. [Consulta: 02 de Abril de 2014].

OMS (1998). Programa de nutrición, familia y reproducción saludable. Obesidad: prevención y gestión de la epidemia mundial. Informe de una consulta de la OMS sobre obesidad. Ginebra: OMS.

Parra, M. (2001). Programa de actividades físicas en la naturaleza y deportes de aventura para la formación del profesorado de segundo ciclo de secundaria. Tesis Doctoral, Granada, Facultad de Ciencias de la Educación.

Pinto, A., Abobeleira, J., Costa, J., Ferreira, N., Pinto y N., Rainho, C. (2009). Vigilancia de la salud de los mayores: Salud Percibida (SF-36). En VI Congreso Nacional FAECAP. Oviedo.

Ramos, P. y Pinto, J.A. (Coord.) (2005). Actividad física y ejercicio en los mayores. Madrid: Instituto de Salud Pública. Comunidad de Madrid. 
Rikli, R. E. y Jones, C. J. (1999). Functional fitness normative scores for community residing older adults, ages 60-94. Journal of Aging and Physical Activity, 7, 162- 181.

Rikli, R. E. y Jones, C.J. (2001). Senior Fitness Test Manual. Champaign IL: Human Kinetics.

Rosenfeld, O. y Tenembaum, G. (1992). Physical fitness in the industrial environment: perceived physical wellbeing benefits. International Journal of Sport Psychology, 23, 227-242.

Ruiz Montero, P. J. y Baena, A. (2009). Grado de influencia de la actividad física sobre los estados de ánimo en mujeres de 60 a 79 años. En Montiel, P., Merino, A., Sánchez, A., Heredia, A. y Salinas, F. (comp.), III Congreso Internacional de Actividad Físico Deportivo para mayores (pp. 876- 884). Málaga: CEDMA.

Schwerdtfeger, A., Eberhardt, R., y Chmitorz, A. (2008). Is there a correlation between everyday-life physical activity and psychological well-being? A methodological study using ambulatory monitoring. Zeitschrift fur gesundheitspsychologie, 1 (16), 2-11.

Ware, J. E. (2000). SF-36 health survey update. Spine, 25, 3130-3139.

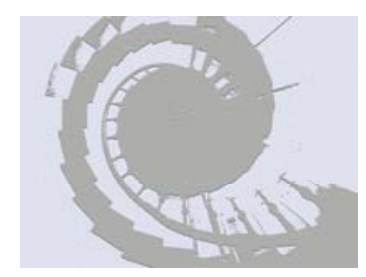

